\title{
COMMUTATION RELATIONS FOR TRUNCATED TOEPLITZ OPERATORS
}

\author{
ISABELlE CHALENDAR AND DAN TIMOTIN
}

\begin{abstract}
For truncated Toeplitz operators, which are compressions of multiplication operators to model subspaces of the Hardy space $H^{2}$, we obtain criteria for commutation relations. The results show an analogy to the case of Toeplitz matrices, and they extend the theory of Sedlock algebras.
\end{abstract}

Mathematics subject classification (2010): 47B32, 47B35, 47B37.

Keywords and phrases: Truncated Toeplitz operators, normal operators, commutation properties.

\section{REFERENCES}

[1] P. R. Ahern and D. N. Clark, Radial limits and invariant subspaces, Amer. J. Math. 92 (1970), 332-342.

[2] A. Baranov, R. Bessonov, And V. Kapustin, Symbols of truncated Toeplitz operators, J. Funct. Anal. 261 (2011), 3437-3456.

[3] A. Baranov, I. Chalendar, E. Fricain, J. Mashreghi, and D. Timotin, Bounded symbols and reproducing kernel thesis for truncated Toeplitz operators, J. Funct. Anal. 259 (2010), 2673-2701.

[4] J. A. Cima, S. R. Garcia, W. T. Ross, And W. R. Wogen, Truncated Toeplitz operators: spatial isomorphism, unitary equivalence, and similarity, Indiana Univ. Math. J. 59 (2010), 595-620.

[5] J. A. Cima, W. T. Ross, And W. R. Wogen, Truncated Toeplitz operators on finite dimensional spaces, Oper. Matrices 2 (2008), 357-369.

[6] D. N. CLARK, One dimensional perturbations of restricted shifts, J. Analyse Math. 25 (1972), 169191.

[7] P. J. Davis, Circulant Matrices, Wiley, New York, 1979.

[8] S. R. Garcia And M. Putinar, Complex symmetric operators and applications, Trans. Amer. Math. Soc. 358 (2006), 1285-1315.

[9] S. R. Garcia AND W. T. Ross, Recent progress on truncated Toeplitz operators, Fields Institute Communications 65 (2013), 275-319.

[10] S. R. Garcia, W. T. Ross, AND W. R. Wogen, Spatial isomorphisms of algebras of truncated Toeplitz operators, Indiana Univ. Math. J. 59 (2010), 1971-2000.

[11] J. B. GaRnett, Bounded Analytic Functions, Springer, New York, 2007.

[12] C. Gu AND L. PATton, Commutation relations for Toeplitz and Hankel matrices, SIAM J. Matrix Anal. Appl. 24 (2003), 728-746.

[13] N. K. NIKOLSKI, Operators, functions, and systems: an easy reading, American Mathematical Society, Providence, RI, 2002.

[14] D. SARASON, Algebraic properties of truncated Toeplitz operators, Oper. Matrices 1 (2007), 491-526.

[15] N. A. SEDlock, Algebras of truncated Toeplitz operators, Oper. Matrices 5 (2011), 309-326.

[16] T. Shalom, On algebras of Toeplitz matrices, Linear Algebra Appl. 96 (1987), 211-226.

[17] E. Strouse, D. Timotin, AND M. ZARRABi, Unitary equivalence to truncated Toeplitz operators, Indiana Univ. Math. J. 61 (2012), 525-538.

[18] B. SZ.-NAGY AND C. FoIAs, Harmonic analysis of operators on Hilbert space, North-Holland Publishing Co., Amsterdam-London, 1970. 